(1985) Pferdeheilkunde 1, 149-153

\section{Beobachtungen zur Entstehung des Sommerekzems}

\author{
P. J. Quinn
}

Department of Farm Animal Clinical Studies, Veterinary College, Ballsbridge, Dublin

\section{Einleitung}

Hautveränderungen durch Ektoparasitenbefall sind bei vielen Tierarten beschrieben. Solche Veränderungen können die Folge direkter mechanischer Schädigung, der Abgabe zytotoxischer oder pharmakologisch wirksamer Substanzen, lokaler Entzündungserscheinungen nach ausgelöster Infektion oder der Sensibilisierung aufgrund der eingebrachten Allergene sein (Walton, 1971). Ektoparasiten sind eine wichtige Ursache für allergische Hautreaktionen, und viele Tierarten werden, nachdem sie wiederholt mit den Sekreten stechender Insekten in Kontakt kamen, ihnen gegenüber sensibilisiert. Es wird weltweit unter lokal unterschiedlichen Bezeichnungen über eine chronisch saisonal rezidivierende Dermatitis bei Pferden berichtet, die durch eine ungewöhnliche Lokalisierung im dorsalen Bereich der Körperoberfläche charakterisiert ist. In einigen Ländern zieht man den Begriff „sweet itch“, d. h. Sommerekzem, oder Mähnen- und Schweifekzem zur Beschreibung dieser oberflächlichen Dermatitis heran. Für die Erscheinungen wurde eine Überempfindlichkeitsreaktion auf stechende Insekten verantwortlich gemacht (Riek, 1972; Mellor und McCaig, 1974; Quinn, Baker und Morrow, 1983). In Australien hat Riek (1954) diese Veränderungen beschrieben, in England waren es Mellor und McCaig (1974) und in Irland Baker und Quinn (1978). Erkrankungen mit ähnlichen klinischen Erscheinungen wurden auf der ganzen Welt geschildert, aber es ist nicht geklärt, ob diese unterschiedlichen klinischen Zustandsbilder eine gemeinsame oder verwandte Ätiologie haben.

\section{Klinik des Sommerekzems}

Das Sommerekzem und die entsprechenden Erkrankungen in anderen Ländern sind durch starken Juckreiz der betroffenen Bereiche charakterisiert. Die Hautschäden, die sich anfangs in der Mähne und am Schweifansatz finden, neigen bei wiederholter Exposition zur Ausdehnung und können auch seitlich an Hals und Schultern und ebenso an der dorsalen Körperoberfläche auftreten (Abb. 1). Im weiteren Verlauf des pathologischen Geschehens kommt es zum Haarausfall an Mähne, Widerrist und Rumpf (Abb. 2). Die Haare brechen, und die Haut zeigt hyperkeratotische Faltenbildung in den erkrankten Bezirken (Abb. 3). In chroni- schen Fällen bleibt die Haut verdickt, trocken, rauh und haarlos. An den betroffenen Körperteilen tritt Hyperästhesie auf (Baker und Quinn, 1978), und die Patienten reiben sich, da die Veränderungen stark jucken, manchmal unter Verlust der Epidermis selbst wund. In schwer traumatisierten Bereichen kann es zu Ulzerationen und Sekundärinfektionen kommen. In gemäßigten Klimazonen, wo die Flugaktivität stechender Insekten unter Umständen im späten Frühjahr beginnt und bis zum Herbst andauert, zeigen die befallenen Pferde möglicherweise bis zum ersten Frost nur geringe Anzeichen von Besserung, wenn keine Behandlung erfolgt. Obwohl die Erscheinungen als Erkrankung in erster Linie Ponys betreffen, können sie auch bei Huntern, Zugpferden, Arabern und Vollblütern auftreten. Die Verbreitung des Sommerekzems in der Pferdepopulation von Großbritannien wurde mit 2 Prozent angegeben (Anon, 1965). Eine Übersicht über Ponys sagt aus, daß 2,79 Prozent der Ponypopulation von Sommerekzem betroffen sind (McCaig, 1973). Bei der Häufigkeit des Auftretens der Krankheit zeigen sich beträchtliche geographische Unterschiede, wobei aus einzelnen Gebieten Berichte vorliegen, daß über 4,6 Prozent der Tiere befallen waren. Eine Veröffentlichung über das Sommerekzem in Israel gab an, daß die Wahrscheinlichkeit, daß ein Pferd an Sommerekzem erkrankt, mit zunehmender Körpergröße abnimmt (Braverman et al., 1983). Nach dieser Studie waren außerdem Hengste empfindlicher als Stuten, und helle Pferde zeigten sich weniger anfällig als dunkle.

\section{Histopathologie der Hautschädigungen beim Sommerekzem}

Die Gewebsveränderungen im Zusammenhang mit dem Sommerekzem ähneln den bei Hunden mit Überempfindlichkeit gegenüber Flohbissen beobachteten (Baker und Flanagan, 1975). Sie sind durch subepidermales Ödem, Infiltration mit eosinophilen Leukozyten in großer Zahl (Baker und Quinn, 1978) und den typischen Erscheinungen einer allergischen Reaktion vom Soforttyp charakterisiert. Die intradermale Injektion wäßriger Extrakte von Culicoides spp. bei erkrankten Pferden rief ein ähnliches histologisches Bild hervor wie bei natürlicherweise auftretenden Schädigungen. Die Reaktionen an der Injektionsstelle setzten innerhalb von 20 Minuten ein, und dies zusammen mit der eosinophilen Infiltration deutet auf eine Beantwortung in Form einer allergischen Reaktion vom Soforttyp hin (Baker und Quinn, 1978).

\section{Ätiologie des Sommerekzems}

Unter den vermuteten Ursachen des Sommerekzems hat man Sonnenlicht, Grasallergie, Bakterien und Pilze, Mineralstoff- und Vitaminmangellagen, Mikrofilarien und Stechfliegen aufgeführt (McCaig, 1975). Es gibt wenig überzeugende Beweise dafür, daß eine Beziehung zwischen den in Betracht gezogenen Wirkstoffen und dieser saisonal bedingten Dermatitis besteht. In zunehmendem Maße werden Stechfliegen als vermutliche Ursache der Erkrankung damit in Zusammenhang gebracht (Mellor und McCaig, 

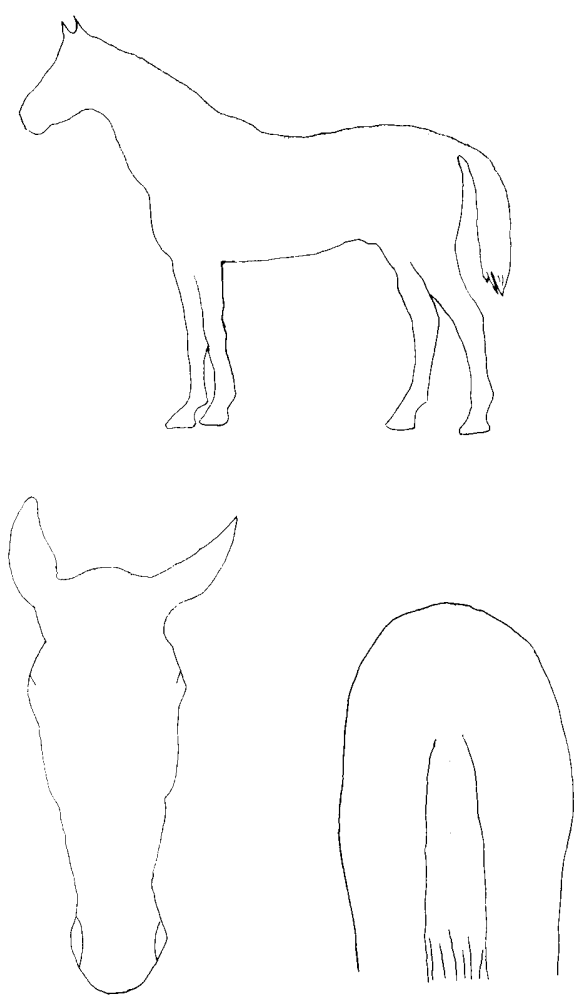

Abb. 1: Verteilung der Sommerekzem-Veränderungen.

1974; Baker und Quinn, 1978; Braverman et al., 1983; Quinn et al., 1983). Riek (1954) berichtete, die allergische Dermatitis bei Equiden trete infolge der sich entwickeln- den Überempfindlichkeit gegenüber den Stichen von Culicoides robertsi auf. In Arbeiten über das Sommerekzem in England wurde die These erstellt, Culicoides pulicaris sei wahrscheinlich die Ursache (Mellor und McCaig, 1974).

Erkrankte Pferde, die eine intradermale Injektion eines sterilen Extrakts von Culicoides, Tabanidae, Stomoxys und Culex erhalten hatten, reagierten stark auf den CulicoidesExtrakt (Quinn et al., 1983). Abb. 4 zeigt die Reaktionen zweier allergischer Pferde, bei denen man die Exposition mit $0,1 \mathrm{ml}$ intradermal verabreichten Insektenextrakten durchgeführt hatte. Im Vergleich mit steriler isotoner Kochsalzlösung (0,85prozentiges Natriumchlorid) zeigten beide Pferde eine starke Reaktion auf den CulicoidesExtrakt, und Pferd $\mathrm{T}$ reagierte in geringerem Maße auch auf andere Extrakte. Bestimmte Culicoides-Arten können stärkere Hautreaktionen hervorrufen als andere (Morrow, 1982). Abb. 5 stellt dies dar. Die Reaktionen auf Culicoides waren bei einer Gruppe von 10 kranken Pferden im Abstand von 20 Minuten und 1 Stunde nach der Exposition wesentlich stärker als bei einer klinisch normalen Gruppe ( $\mathrm{p}<0,025)$ (Quinn et al., 1983).

Die Ergebnisse aktiver Hauttests bei Pferden mit Sommerekzem wiesen darauf hin, daß die Reaktion diejenige einer Allergie vom Soforttyp ist (Baker und Quinn, 1978). Die passive Übertragung von Serum von erkrankten Pferden auf gesunde Pferde bestätigte, daß die Reagibilität mit Serum übertragbar ist (Quinn et al., 1983).

Abb. 6 zeigt die Reaktion eines kranken Pferdes (Pferd GE) auf die Exposition mit intradermal verabreichten Insektenextrakten. Das Serum dieses Pferdes wurde dann auf ein gesundes Pferd übertragen, indem man an verschiedenen Stel-

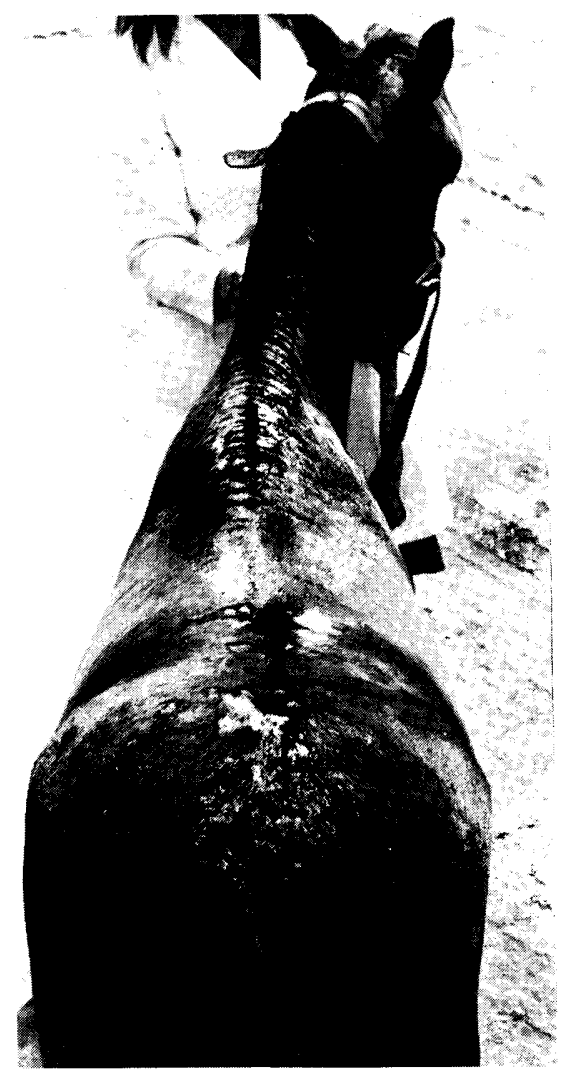

4 Abb. 2: Veränderungen beim Sommerekzem dorsal am Hals, am Widerrist und im Lumbosakralbereich eines schwer erkrankten Pferdes.

V Abb. 3: Hyperkeratotische Faltenbildung der Haut im Mähnenbereich eines Pferdes mit chronischer Erkrankung.

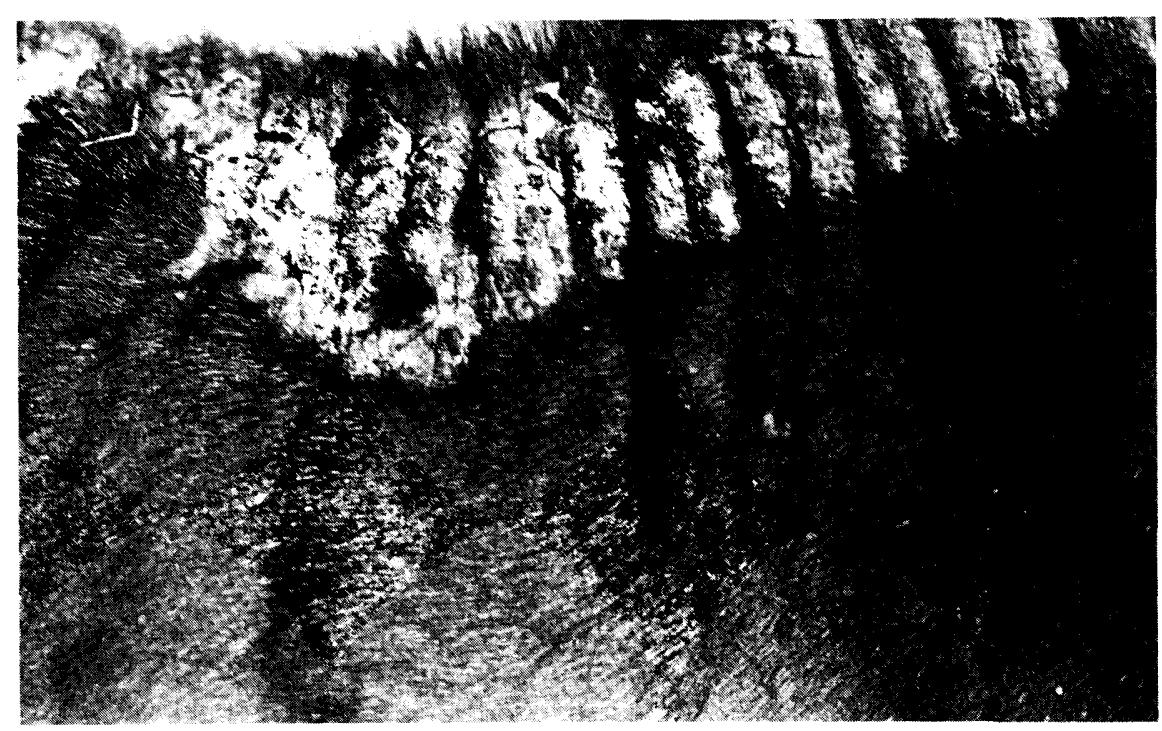




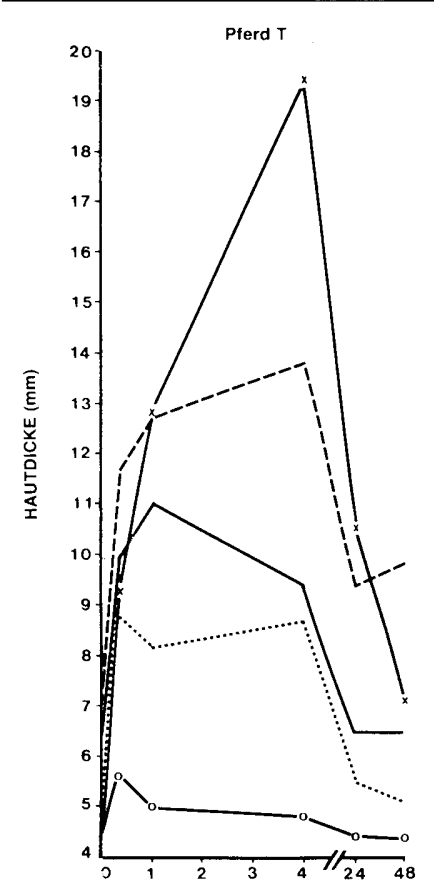

ZEIT NACH DER EXPOSITION (Stunden)

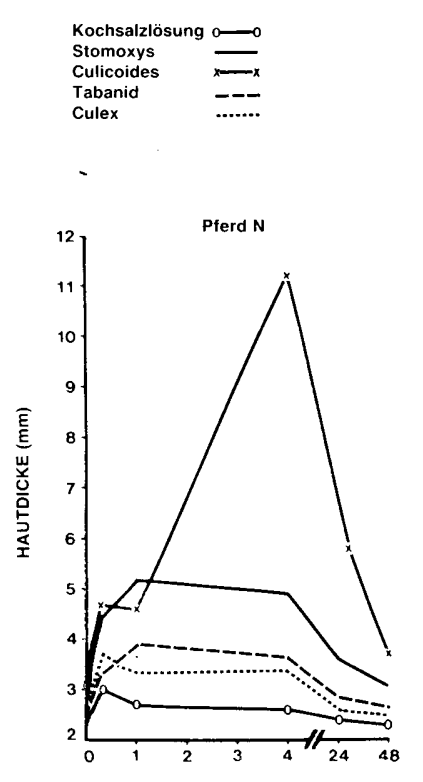

ZEIT NACH DER EXPOSITION (Stunden)

Abb. 4: Intradermale Exposition zweier kranker Pferde mit Stechflie genextrakten und Kochsalzlösung. Die Reaktionen wurden an Hand von Messungen der Hautfaltendicke vor und 20 Minuten, 1, 4, 24 und 48 Stunden nach der Exposition aufgezeichnet.

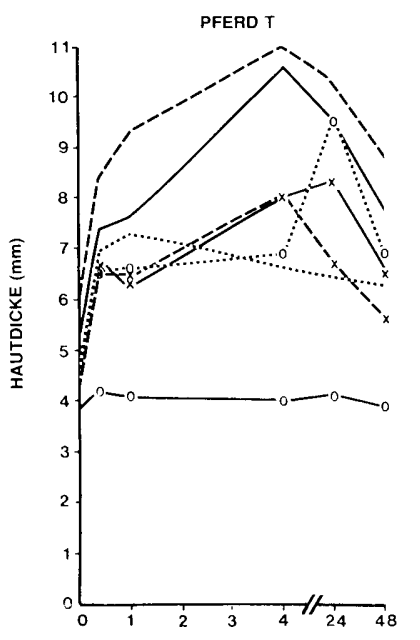

ZEIT NACH DER EXPOSITION (Stunden)

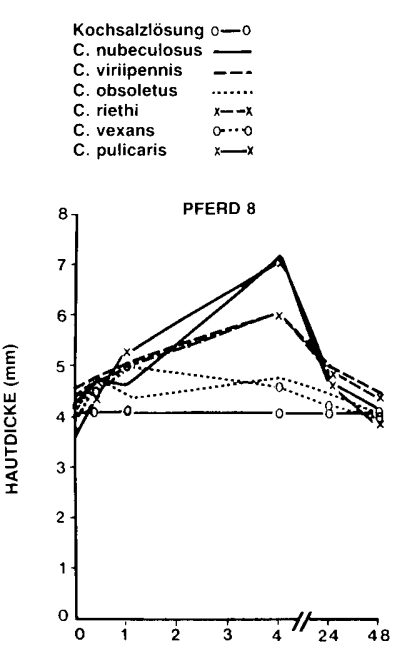

ZEIT NACH DER EXPOSITION (Stunden)

Abb. 5: Intradermale Exposition eines kranken (Pferd T) und eines gesunden (Pferd 8) Pferdes mit Extrakten verschiedener Culicoides-Arten und isotoner Kochsalzlösung. Die Reaktionen wurden an Hand von Messungen der Hautfaltendicke vor und 20 Minuten, 1, 4, 24 und 48 Stunden nach der Exposition aufgezeichnet.

len $0,2 \mathrm{ml}$ intradermal injizierte. Nach Latenzzeitspannen von 24, 48 und 72 Stunden wurden an jeder Stelle zur Exposition Stechfliegenextrakte oder sterile Kochsalzlösung eingebracht. Die Ergebnisse bestanden laut Bericht in Hautfaltenverdickung und ödematisierten Bereichen. Abb. 7 macht deutlich, daß die übertragenen Serum-Antikörper mindestens 72 Stunden an der Haut des Empfängers fixiert blieben und daß die stärkste Reaktion sowohl in

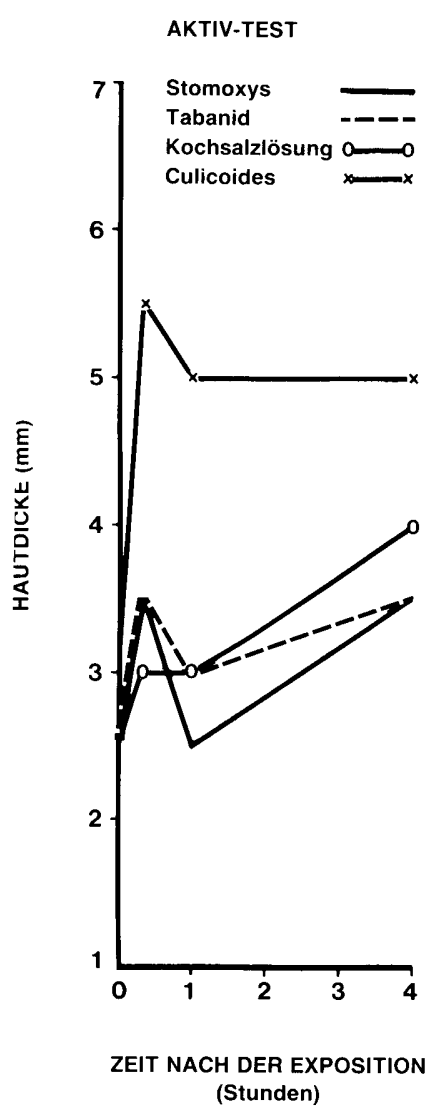

Abb. 6: Intradermale Exposition eines kranken Pferds (Pferd GE) mit Stechfliegenextrakten und isotoner Kochsalzlösung.

Form von Hautfaltenverdickung als auch von Ödematisierung an Stellen von Exposition mit Culicoides-Extrakt ablief.

Obwohl bei den Experimenten mit passiver Übertragung einige Unterschiede unter den kranken Pferden zu beobachten waren, lief bei dem gesunden Empfänger an der passiv sensibilisierten Stelle immer eine stärkere Reaktion auf die Culicoides-Extrakte ab als auf die anderen getesteten Insektenextrakte.

\section{Bevorzugte Körperregionen - mögliche Erklärungen}

Für die ungewöhnliche Verteilung der Hautschäden beim Sommerekzem wurden verschiedene Faktoren verantwortlich gemacht. Man hat auf drei verschiedenen Wegen die Lokalisation der Veränderungen bei erkrankten Pferden zu erklären versucht: (1) an einem Lockpferd wurden Landeplätze und Stichstellen von Culicoides untersucht; (2) die je nach Körperregion unterschiedliche Reaktion auf die intradermale Exposition mit Stechfliegenextrakten wurde verglichen, und (3) die Verteilungsmuster der Mastzellen in der Pferdehaut wurden studiert.

Von den über 10000 von einem Lockpferd angezogenen Culicoides stachen 40 Prozent (Townley, Baker und Quinn, 1984). Die Mehrheit der Insekten, die eine volle Blutmahlzeit nahmen, saugte an der Mähne und im distalen Gliedmaßenbereich. Gebiete entlang der dorsalen Mittellinie wa- 


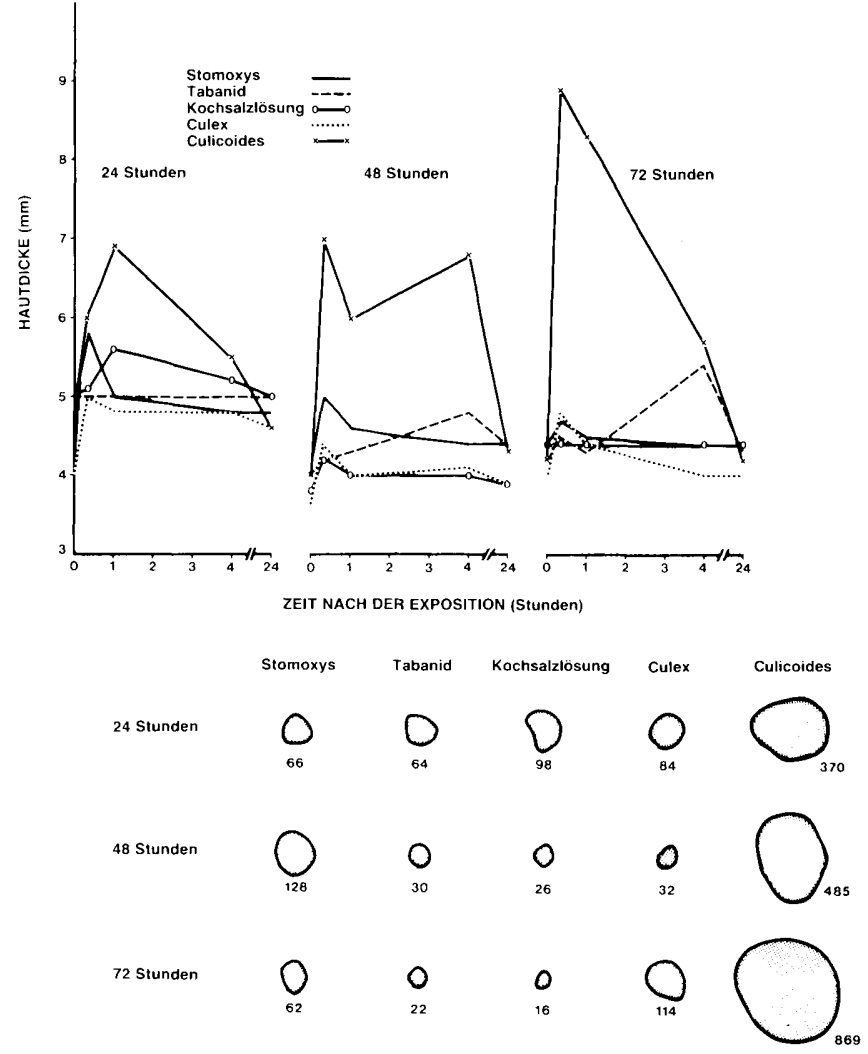

Abb. 7: Passive Übertragung des Serums eines allergischen Pferdes (Pferd GE) auf Stellen in der Haut eines normalen Pferdes und nachfolgende Exposition dieser Stellen nach Latenzzeitspannen von 24, 48 und 72 Stunden mit Stechfliegenextrakten. Der untere Teil der Abbildung zeigt die ödematisierten Bereiche bei dem passiv sensibilisierten Pferd nach 1 Stunde für die 24stündige Latenzzeit und nach 20 Minuten für die 48- und 72stündige Latenzzeit. Die Zahlen geben die tatsächliche Ödemreaktion an $\left(\mathrm{mm}^{2}\right)$.

ren die bevorzugten Stichstellen einiger Spezies: C. punctatus, C. nubeculosus und C. pulicaris saugten in erster Linie an den Prädelektionsstellen des Sommerekzems. In beträchtlichem Maß befielen sie jedoch auch den unteren $\mathrm{Ab}$ schnitt der Beine, wo die Schädigungen des Sommerekzems nicht auftreten.

Aktive Hauttests mit Stechfliegenextrakten in verschiedenen Körperbereichen eines kranken und eines klinisch gesunden Pferdes führten am Widerrist und am Schweifansatz zu den stärksten Reaktionen (Morrow, 1982).

Mastzellzählungen in Proben bei über 30 Hautgebieten von sieben normalen Pferden ließen kein klares Muster erkennen, aber öfter sind diese Zellen in größerer Zahl entlang der dorsalen Mittellinie vertreten (Morrow, 1982).

\section{In Culicoides-Extrakten auftretende Allergene}

Blutsaugende Parasiten, die ein Säugetier als Wirt für eine Blutmahlzeit befallen, sensibilisieren oft das Wirtstier, da die Speichelsekrete blutsaugender Insekten gewöhnlich ein oder mehrere wirksame Allergene enthalten (Nelson, Bell, Clifford und Keirans, 1977).

Die Fraktionen eines Culicoides-Extraktes, die mit Hilfe von Gelfiltration und präparativer isoelektrischer Fokussierung aufgetrennt worden waren, wurden an zwei Pfer- den mit Sommerekzem auf ihre allergene Wirkung untersucht. Bei der Anwendung von Flachbett-Elektrophorese waren auf Coomassie-blau gefärbten Papierabzügen der aufgetrennten Fraktionen über 20 separate Banden sichtbar (Morrow, 1982). Bei mindestens vier von ihnen konnte man eine allergene Wirkung nachweisen.

\section{Mediatoren mit Beteiligung an allergischen Hautreaktionen beim Pferd}

Nach versuchsweiser intradermaler Verabreichung von Histamin, Serotonin und Bradykinin in verschiedenen Konzentrationen an sechs Pferde waren die Reaktionen, die Histamin hervorrief, stärker als die durch die beiden anderen Mediatoren ausgelösten (Morrow, 1982). Zwei Antihistaminika, Tripelenaminhydrochlorid und Promethazinhydrochlorid, erwiesen sich beide als wirksame Antagonisten bei Hautreaktionen nach injizierten Stechfliegenextrakten und bei Histamin selbst. Diese Befunde lassen vermuten, daß Histamin der Hauptmediator der allergischen Reaktion vom Soforttyp bei dieser Spezies ist. Hanna, Eyre, Wells und McBeath (1982) haben die biochemischen Grundlagen der Allergie beim Pferd besprochen.

\section{Behandlung und Prophylaxe des Sommerekzems}

Man hat eine Reihe unterschiedlicher Behandlungsmaßnahmen durchgeführt, um die mit dem Juckreiz verbundenen Schädigungen möglichst gering zu halten. Es wurden Kortikosteroide und Antihistaminika empfohlen (McCaig, 1975). Wirkstoffe mit höherer Spezifität als Kortikosteroide erscheinen besser geeignet. Antihistaminika gewähren kurzfristige Milderung der infolge des Juckreizes auftretenden Symptome.

Es bestehen Anhaltspunkte dafür, daß einige allergische Erkrankungen unter Umständen von Erbfaktoren beeinflußt sind, und möglicherweise gehört das Sommerekzem zu dieser Kategorie (Frost, 1974; McCaig, 1975).

Die beste Maßnahme zur Verhütung des Sommerekzems bleibt die Aufstallung erkrankter Pferde in fliegendichten Ställen während der Flugzeiten von Culicoides. Die Zeit des Weidegangs darf nicht bis in die Dämmerung hineinreichen, besonders an windstillen Abenden. Empfängliche Pferde werden am besten vom späten Nachmittag bis zum folgenden Morgen während der Perioden der Flugaktivität der Fliegen im Stall gehalten. Als Alternative kann man versuchen, insektenvertreibende Mittel wie synthetische Pyrethroide anzuwenden. Sie müssen häufig aufgebracht werden, gewöhnlich im wöchentlichen Abstand oder bei feuchter Witterung öfter.

Beim Menschen wird zunehmend die Desensibilisierung als mögliche Langzeitmaßnahme bei der Behandlung vieler allergischer Reaktionslagen herangezogen (Patterson, 1979; Hendrix, Patterson, Zeiss und Suszko, 1981). Die chemische Polymerisierung von Culicoides-Allergenen bietet vielleicht einen zukünftigen Weg, die Bildung von „Block“-Antikörpern bei kranken Pferden zu induzieren, und schafft so eine befriedigendere Möglichkeit, diese rezidivierende saisonale Dermatitis in den Griff zu bekommen. 


\section{Literatur}

Anon. (1965): British Equine Veterinary Association survey of equine diseases, 1962 bis 1963 . Veterinary Record 77, 528-537.

Baker, K. P. and Flanagan, J. O. (1975): Hypersensitivity of dog skin to fleas, a clinical report. Journal of Small Animal Practice 16, 317-327.

Baker, K. P. and Quinn, P. J. (1978): A report on clinical aspects and histopathology of sweet itch. Equine Veterinary Journal 10, 243-248.

Braverman, Y., Ungar-Waron, H., Frith, K., Adler, H., Danieli, Y., Baker, K. P. and Quinn, P. J. (1983): Epidemiological and immunological studies of sweet itch in horses in Israel. Veterinary Record 112, 521-524.

Frost, R. D. I. (1974): Sweet itch. Veterinary Record 94, 28.

Hanna, C. J., Eyre, P., Wells, P. W. and McBeath, D. G. (1982): Equine Immunology 2: Immunopharmacology - biochemical basis of hypersensitivity. Equine Veterinary Journal 14, 16-24.

Hendrix, S. G., Patterson, R., Zeiss, C. R. and Suszko, L. M. (1981): Further studies on the safety of polymerized antigens for immunotherapy. Journal of Allergy and Clinical Immunology 67, 124-128.

Mellor, P. S. and McCaig, J. (1974): The probale cause of sweet itch in England. Veterinary Record 95, 411-415.

McCaig, J. (1973): A survey to establish the incidence of sweet itch in ponies in the United Kingdom. Veterinary Record 93, 444-446.

McCaig. J. (1975): Recent thoughts on sweet itch. Veterinary Annual, 15th Edition. Edited by C.S.G. Grunsell and F.W.G. Hill. Wright and Sons, Bristol. pp. 204-206.

Morrow, $A$. N. (1982): An investigation of allergic reactions in horse skin to biting insects. Ph. D Thesis, University College Dublin.

Nelson, W. A., Bell, J. F., Clifford, C. M. and Keirans, J. E. (1977): Interactions of ectoparasites and their hosts. Journal of Medical Entomology $13,389-428$.
Patterson, $R$. (1979): Clinical efficacy of allergen immunotherapy. Journal of Allergy and Clinical Immunology 64, 155-158.

Quinn, P. J., Baker, K. P. and Morrow, A. N. (1983): Sweet itch: responses of clinically normal and affected horses to intradermal challenge with extracts of biting insects. Equine Veterinary Journal 15, 266-272.

Riek, S. F. (1954): Studies on allergic dermatitis (Queensland Itch) of the horse; the aetiology of the disease. Australian Journal of Agricultural Research 5, 109-129.

Riek, R. F. (1972): Queensland Itch. In: Equine Medicine and Surgery, Second Edition. Edited by E. J. Catcott and J. F. Smithcors. American Veterinary Publications, Illinois. pp. 233-236.

Townley, P., Baker, K. P. and Quinn, P. J. (1984): Preferential landing and engorging sites of Culicoides species landing on a horse in Ireland. Equine Veterinary Journal 16, 117-120.

Walton, G. S. (1971): Allergic responses involving the skin of domestic animals. Advances in Veterinary Science and Comparative Medicine $15,201-240$.

\section{P. J. Quinn}

Department of Farm Animal Clinical Studies

Faculty of Veterinary Medicine

Ballsbridge, Dublin 4

Die Forschungen, über die der Autor und seine Mitarbeiter K. P. Baker und A. N. Morrow in dieser Veröffentlichung berichtet haben, wurden mit finanzieller Unterstützung einer Kreditgesellschaft durchgeführt. 